\title{
Brake Operation and Palmar Perspiration Reflect Older Adult Drivers' Ability to Predict Hazards: Driving Simulation Research
}

\author{
Jun Iwanami ${ }^{1}$, Masayoshi Kobayashi ${ }^{1}$, Akira Sagari ${ }^{1}$, \\ Tsutomu Sasaki ${ }^{2}$, Hideya Momose ${ }^{3}$, Toshio Ohhashi ${ }^{4}$ \\ ${ }^{1}$ Graduate School of Medicine, Shinshu University, Nagano, Japan \\ ${ }^{2}$ Hokkaido Chitose College of Rehabilitation, Hokkaido, Japan \\ ${ }^{3}$ SKINOS Co., Ltd., Nagano, Japan \\ ${ }^{4}$ Department of Innovation of Medical Health Sciences Research, Shinshu University School of Medicine, Nagano, Japan
}

\begin{abstract}
Objective: Motor accidents caused by older drivers have been increasing and may result from a decline in cognitive functioning and delay in hazard perception. This study examined how brake operation and palmar perspiration indicate hazard predictive ability of older drivers in a driving simulation.

Method: We compared brake operation performance, palmar sweating response, and skin potential reflex responses in healthy older adults $(n=43)$ and healthy young adults $(n=36)$ during hazard and hazard prediction scenes in a driving simulator.

Results: In the hazard scene, both groups displayed rapid brake operation and skin potential reflex responses. In the hazard prediction scene, all young adults braked consistent with simulation footage, but $46.5 \%$ of older adults failed to brake. Palmar sweating response was greater $(p<0.01)$ in older adults who braked compared to older adults who did not. In those who failed to break, palmar sweating response was lower than the overall average observed in the older adult group, suggesting that non-operating group members lacked a sense of tension.

Conclusion: Cognitive processes of hazard perception and hazard prediction may facilitate the observed increase in palmar sweating response. Brake operation monitoring and palmar sweating response measurement appear to be useful for evaluating hazard perception ability in a driving simulator.
\end{abstract}

Keywords: simulated driving, older drivers, hazard perception, brake operation, palmar perspiration

(Asian J Occup Ther 16: 1-9, 2020)

\section{Introduction}

In Japan, an increasingly aging population has been accompanied by an increase in automobile accidents. In 2016, drivers aged 75 and older caused $13 \%$ of all fatal accidents [1]. Such accidents are associated with agerelated cognitive decline $[2,3]$; thus, cognitive evaluations are now mandatory to renew the licenses of drivers over 75. According to the National Police Agency,

Received: 26 August 2018, Accepted: 27 October 2019

Corresponding to: Masayoshi Kobayashi, Ph.D., Graduate School of Medicine, School of Health Science, Shinshu University, 3-1-1, Asahi, Matsumoto 390-8621, Japan

e-mail: mkobaya@shinshu-u.ac.jp

C2020 Japanese Association of Occupational Therapists enforcement of this new law resulted in 1,120,000 individuals undergoing cognitive function testing between March and September 2017. Examination resulted in 300,000 aging drivers to be diagnosed with possible dementia; $697(0.23 \%)$ had their licenses suspended or revoked. This implies that it is difficult to determine driving ability via a written cognitive function test administered during license renewal.

Pre-driving (i.e., driving simulation) and practical driving (i.e., road driving) tests can be incorporated into comprehensive evaluations aiming to help disabled individuals resume driving. The Trail Making Test (TMT) - assessing attention, visual search, information processing speed, and executive function-is used for neurophysiological aspects of pre-driving evaluations [4-6] and has been proven effective [7]. The Mini-Men- 
tal State Examination (MMSE) also measures cognitive function; it may be administered when evaluating an older driver. A study using standardized road driving evaluations, neurophysiological examinations, and medical tests to assess Alzheimer's patients found that the MMSE usefully predicted older adults' driving ability [8]. However, others have suggested that cognitive function tests, such as the MMSE, cannot adequately determine the driving ability of older adults with earlystage dementia [9]. Pre-driving evaluations of this nature - even those that include neurophysiological examinations - illustrate that defining one's driving ability is difficult, and costs and accident risks limit the applicability of driving evaluations [10]. Additionally, among older adults, cessation of driving has been associated with a worsening of depressive symptoms [11, 12] and the shrinking of one's social network [13]. Therefore, new research is needed that examines diagnostic methods under which older adults and individuals with cranial trauma may resume or continue driving.

Hazard perception (HP), the process of responding to dangerous conditions with traffic accident potential [14], is a critical ability for safe automobile operation; it is "the ability to read the road and anticipate forthcoming events" $[15,16]$. Further, research indicates that HP is related to processes such as "anticipation" and "surprise" [14, 17]. HP paradigms have been used in evaluations wherein several minutes of driving footage are played for participants who must identify hazardous events as they occur [18]. However, these methods do not assess participants' real-time behavior in response to hazards. Consequently, it is imperative that evaluations of automobile driving ability include tests that examine the entire chain of processes from HP to vehicle operation.

The palmar sweating response (PSR) and electrodermal activity (EDA) occur when humans are surprised or perceive hazards. PSR is an indicator of "mental sweating" or "emotional sweating" [19]. It can closely follow emotional changes like tension and surprise [2022]. The PSR can also be elicited by situations in which one "steels oneself" in response to unfamiliar situations or by higher-order neurological activity, like mental math $[21,22]$. It reflects activity of the dermal sympathetic nerves, and while it is subject to modification by the cerebral cortex, the central mechanism is closely related to the limbic system [23, 24]. The amygdala, anterior cingulate gyrus [25], and insula [26] are involved in this response. In contrast, EDA reflects activity of the eccrine sweat glands and can be broadly differentiated into skin potential activity (SPA) and skin conductance activity (SCA) based on the methods used to measure it [27-29]. SPA is often measured via the skin potential reflex (SPR), whereas SCA is measured via galvanic skin response (GSR) or skin conductance response (SCR).

One study measured participants' GSR during driving and found that it reflected their level of emotional tension during driving tasks [30]. Further, research measuring drivers' SCR found a strong correlation $(r=0.95)$ between driving SCR and brake pressure, with SCR occurring 0.2 seconds prior to accelerator release and 1.9 seconds prior to braking [31]. In a study showing video clips of driving, police drivers rapidly identified hazards, and their SCRs were significantly greater than those of novice drivers [20]. Research that involved playing HP video clips to three experimental groups of driverstraining drivers, novice drivers, and experienced drivers - showed that the SCR of experienced drivers was twice that of novice drivers and three times that of training drivers, suggesting that HP is a skill learned through driving experience [32]. The above results indicate that PSR and EDA are experimental measures that can appropriately evaluate drivers' HP ability.

We developed a computer-based driving simulator apparatus [33] to assess drivers' HP abilities. Actual automobile parts were used for the steering wheel, accelerator, and brake pedal, which were connected to a spring-based system to provide appropriate resistance and feedback. Steering wheel, accelerator, and brake pedal responses were measured using a potentiometer connected to a computer via an analog-to-digital (A/D) converter. These responses were recorded alongside the output signals of a perspiration meter (SKINOS SKN2000) and a skin potentiometer (SKINOS SPN-01; Fig. 1).

Pre-recorded digital driving footage was played on an LCD monitor. Participants were instructed to perform appropriate mock driving operations using the testing apparatus. The footage shown to participants had an approximate field of view of $90^{\circ}$ and was a five-minutelong, $1 \mathrm{~km}$ course through narrow residential streets. An automatic car was used in the recording. The maximum recorded speed was $40 \mathrm{kph}$ with an average speed of 12 $\mathrm{kph}$. The footage contained scenes requiring participants to predict hazards, such as "jogger approaches from the opposite direction" and sudden hazard scenes, such as "pedestrian runs out from a side street." A green-arrow blinker synced with the footage, indicating the direction participants would need to turn at intersections (Fig. $1-a)$.

This driving simulator employed only real-world footage to maintain the reality of visually perceived information. However, the steering wheel, accelerator, and brake pedal were not linked to the footage itself. Therefore, even if the brake pedal were to be depressed, 

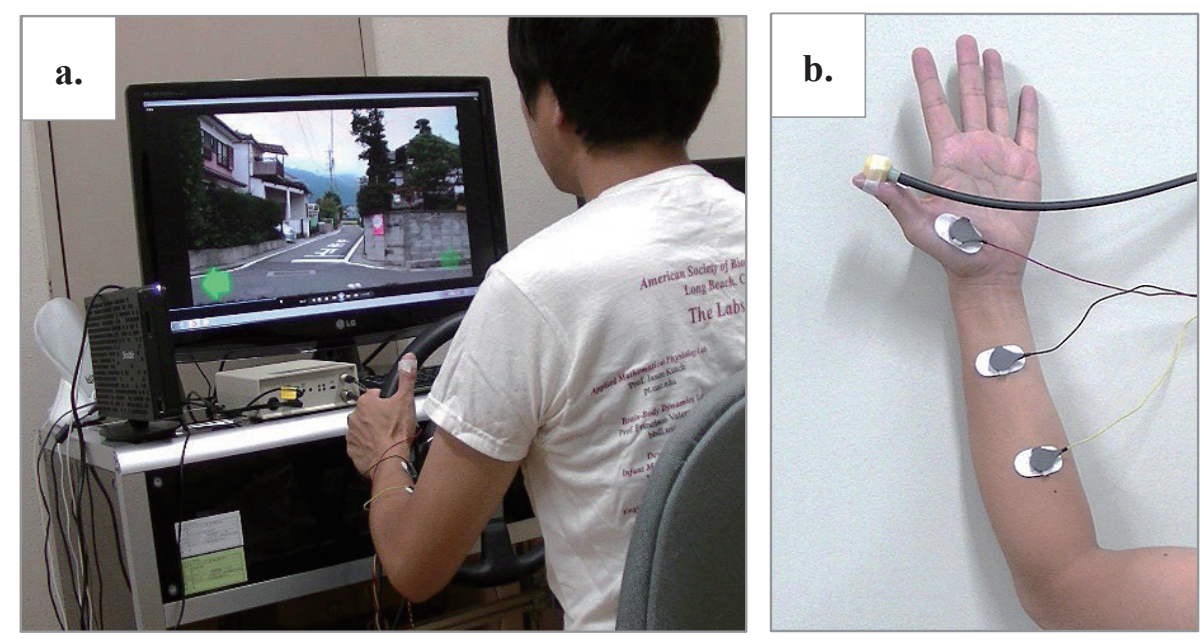

Fig. 1. Driving Simulation Test.

(a) The subject was asked to operate the steering wheel, accelerator, and brake according to the scene displayed on the screen.

(b) PSR was measured by placing a sweating meter probe on the palm of the subject's left thumb. SPR was measured by bipolar induction, in which a skin electrometer electrode was applied to the subject's left forearm and left thumb ball, and a reference electrode was placed between the electrodes.

the car in the footage would not slow. Participants assumed the role of the vehicle's driver and operated the steering wheel, accelerator, and brake consistent with the movements seen on screen. PSR, SPR, and brake operation responses matched to hazard scenes in this type of driving simulation test are largely similar across participants [33]. This study aimed to use a driving simulation test to compare the PSR, SPR, and brake operation performance of older and young adult drivers during hazard and hazard prediction scenes.

\section{Material and Methods}

\section{Participants}

Older adults above the age of $60(n=43 ; 18$ men, 25 women; age range $60-85 ; M_{\text {age }}=69.8$ years, $S D=5.7$ ) and young adults $(n=36 ; 8$ men, 28 women; age range $19-36 ; M_{\text {age }}=21.3, S D=2.8$ ) provided written informed consent before participating in this experiment. The older adult group comprised healthy individuals attending health classes in the Matsumoto region. The older adult group had an MMSE of 24 or higher with no observed decline in cognitive function. The older adults drove daily with 10 plus years of experience each. The young adult group comprised primarily college students and all had a driver's license, but their driving experience was within a few years except for one. No one drove daily.

\section{Experimental Design}

We defined events in the simulation footage of driving through narrow residential streets as specific types of scenes. The "hazard prediction scene" was where the road narrowed with poor visibility, near a fence and hedge, that required depressing the brake and slowing before making a left turn at a T-junction. The sudden emergence of a pedestrian from a side street to the right while the driver was proceeding straight down an alleyway was termed the "hazard scene". For five seconds from the start of both scenes, we checked for the presence or absence of brake operation and summarized the results in a frequency distribution table. Then we compared the PSRs of the group that activated brakes with the group that did not and examined factors that affected the presence or absence of brake operation. Finally, we compared the latencies of brake operation and SPR responses of the two driver groups during the hazard and hazard prediction scene.

\section{Procedure}

The temperature of the experiment was $23-26^{\circ} \mathrm{C}$. PSR elicited by the driving simulation test was measured using a sweating meter probe affixed to the palmar side of the participant's left thumb, where the moisture content of $1 \mathrm{~cm}^{2}$ of skin was measured via the ventilation capsule method. SPR was measured with bipolar leads using a skin potentiometer with electrodes affixed to the participant's left forearm, the ball of the left thumb, and a reference electrode placed in between (Fig. 1-b).

Before the driving simulation test, each participant practiced mock driving in the simulator using footage prepared specifically for practice use. After confirming that the participant could correctly operate the steering wheel, accelerator, and brake pedal, we played the test 
footage at a sound pressure of $50-60 \mathrm{~dB}$. The PSR and SPR responses, as well as the analog signals from the steering wheel, accelerator, and brake pedal, were recorded at a sampling frequency of $100 \mathrm{~ms}$ after $\mathrm{A} / \mathrm{D}$ conversion. This research complied with the tenets of the Declaration of Helsinki and was conducted with the approval of the ethics board of the Shinshu University School of Medicine (approval code 2073).

\section{Data Analyses}

Brake operation was tracked for five seconds immediately following the appearance of either the hazard or hazard prediction scene on the screen. Participants who successfully operated their brakes were placed in the "operation group" and those who did not were placed in the "non-operation group." For the operation group, the time from the start of the hazard or hazard prediction scene to the appearance of a response waveform was termed brake response latency (Fig. 2-c). PSR volume was evaluated as the mean reaction volume over the five seconds immediately following the beginning of the hazard or hazard prediction scene (Fig. 2-a). SPR response latency was evaluated as the time between the start of the hazard or hazard prediction scene to the appearance of a spike-like negative wave (Fig. 2-b). The data of participants who did not show a clear negative spike in their SPR waveforms were regarded as missing and were excluded from analyses.

We confirmed the independence of the frequency distributions of the operation and non-operation groups using a chi-square test. Intergroup comparison of braking, PSR, and SPR response was evaluated with a student's $t$-test. Multivariate logistic regression analysis with forced entry was used to determine the predictors of brake operation. The Hosmer-Lemeshow test was used to examine the goodness-of-fit of this model, and the significance threshold was set at $p>0.05$, showing an acceptable fit. All statistical analyses were performed with IBM SPSS Statistics for Windows release 25 (IBM Japan Ltd., Tokyo, Japan). All tests had a significance level of $p<0.05$.

\section{Results}

\section{Response Waveforms Obtained During Driving Simula- tion Test}

Figure 3 shows examples of response waveforms obtained. In scenes with predictable hazards, and scenes where the participant had to suddenly react to avoid hazards, PSR and SPR nearly synchronized responses of steering wheel and brake pedal operation were observed. In stop scenes, during which the brake pedal was continuously depressed, a decrease in PSR and SPR response

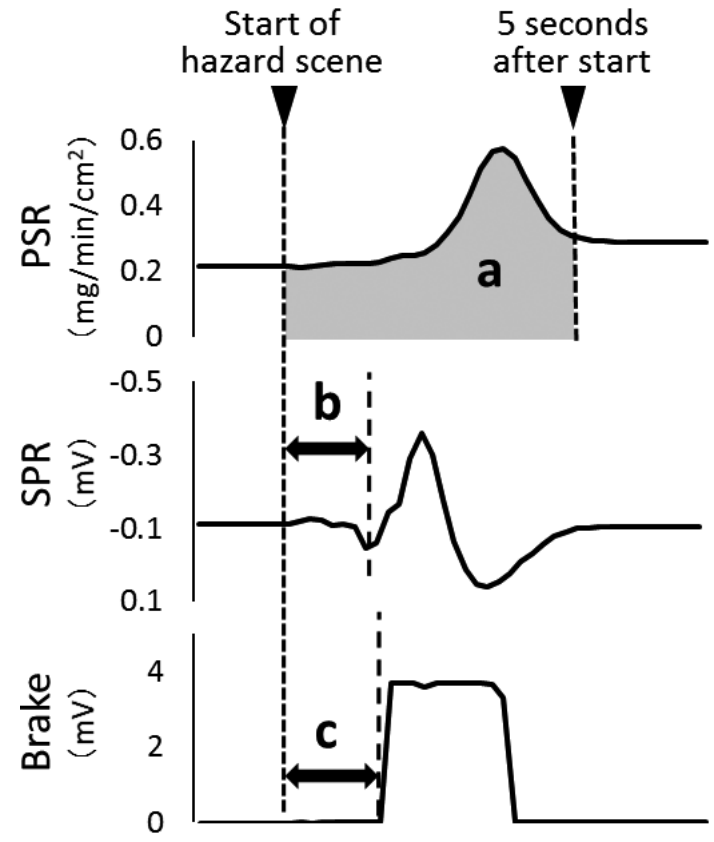

Fig. 2. Measurement Method of PSR Volume, SPR Latency, and Brake Latency.

$\mathrm{PSR}=$ palmar sweating response $; \mathrm{SPR}=$ skin potential reflex; Brake $=$ brake operation.

was seen. SPR also tended to occur a few seconds faster than PSR.

\section{Brake Operation and PSR Volume}

The presence or absence of brake operation in the hazard and hazard prediction scenes is summarized in Table 1. In the hazard prediction scene, 23 participants from the older adult group were placed in the operation group and 20 in the non-operation group. In contrast, all participants from the young adult group were placed in the operation group. The independence of these frequency distributions was confirmed by a chi-square test $(p<0.001)$. In the hazard scene, all participants operated their brakes.

Table 2 displays the PSR reaction volumes of the older and young adult groups in the hazard and hazard prediction scenes. The following PSR volumes were observed $\left[\mathrm{mg} / \mathrm{min} / \mathrm{cm}^{2}\right]$ - hazard prediction scene: older adults $M=0.38(S D=0.26)$, young adults $M=0.20(S D$ $=0.22)$; hazard scene: older adults $M=0.40(S D=0.24)$, young adults $M=0.25(S D=0.28)$. In both scenes, the amount of PSR was significantly higher in the older adult group. In the young adult group, the amount of PSR was significantly higher in the hazard scene than in the hazard prediction scene, but the PSR volume of the elderly adult group was not significantly different between the hazard prediction scene and the hazard scene.

We compared the PSR volumes of the operation 


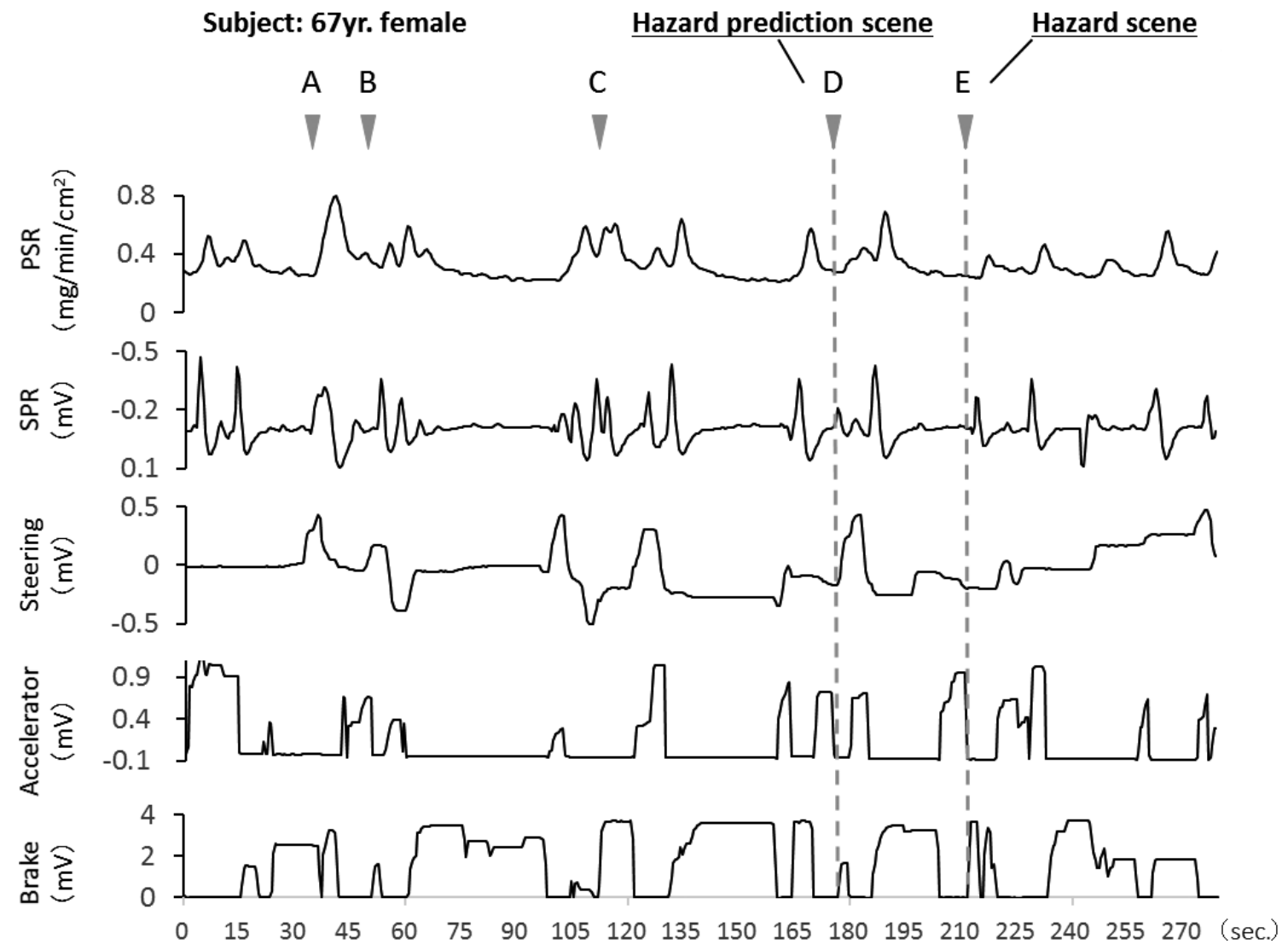

Fig. 3. Changes in PSR, SPR, and Reaction Volume of Steering Wheel, Accelerator, and Brake During Driving Simulation Test. [A] Jogger approaches from the opposite direction; [B] Vehicle approaches from the opposite direction; [C] Participant's vehicle overtakes a bicycle; [D] Participant's vehicle turns left at T-junction (hazard prediction scene); [E] Pedestrian runs out from a side street (hazard scene).

Table 1 Participant's Brake Operation in Hazard Prediction Scene and Hazard Scene.

\begin{tabular}{|c|c|c|c|c|c|}
\hline & Group & $\begin{array}{c}\text { Operation Group } \\
n \\
(\%)\end{array}$ & $\begin{array}{c}\text { Non-Operation Group } \\
n \\
(\%)\end{array}$ & $\chi^{2}$ & $p$ \\
\hline \multirow{2}{*}{ Hazard Prediction Scene } & $\begin{array}{c}\text { Older Adult } \\
n=43\end{array}$ & $\begin{array}{c}23 \\
(53.5)\end{array}$ & $\begin{array}{c}20 \\
(46.5)\end{array}$ & \multirow{2}{*}{22.4} & \multirow{2}{*}{$0.000^{*}$} \\
\hline & $\begin{array}{c}\text { Young Adult } \\
n=36\end{array}$ & $\begin{array}{c}36 \\
(100)\end{array}$ & $\begin{array}{c}0 \\
(0)\end{array}$ & & \\
\hline \multirow{2}{*}{ Hazard Scene } & $\begin{array}{c}\text { Older adult } \\
n=43\end{array}$ & $\begin{array}{c}43 \\
(100)\end{array}$ & $\begin{array}{c}0 \\
(0)\end{array}$ & \multirow{2}{*}{-} & \multirow{2}{*}{-} \\
\hline & $\begin{array}{c}\text { Young adult } \\
n=36\end{array}$ & $\begin{array}{c}36 \\
(100)\end{array}$ & $\begin{array}{c}0 \\
(0)\end{array}$ & & \\
\hline
\end{tabular}

Note. Comparison of Operation Group and Non-Operation Group was conducted using the $\chi^{2}$ test. $* p<.01$.

$(n=23)$ and non-operation $(n=20)$ subgroups of the older adult group in the hazard prediction scene. PSR volumes $\left(\mathrm{mg} / \mathrm{min} / \mathrm{cm}^{2}\right)$ were operation group $M=0.48$ $(S D=0.19)$; non-operation group $M=0.31(S D=0.16)$. The PSR volume of the operation group was significantly larger $(p=0.004)$.
Multivariate logistic regression analysis with forced entry was conducted to investigate the factors related to brake operation by the older adults in the hazard prediction scene. Brake operation was used as the dependent variable. The independent variables included age, gender, and PSR. There was a statistically significant 
Table 2 Comparison of Palmar Sweating Response Volume of Older and Young Adult Groups.

\begin{tabular}{ccccc}
\hline & Older Adult Group & Young Adult Group & & \\
& $n=43$ & $n=36$ & $t$ & $p$ \\
& $M(S D)$ & $M(S D)$ & & \\
\hline Hazard prediction scene & $0.38(0.26)$ & $0.20(0.22)$ & 3.34 & $0.001^{* *}$ \\
Hazard scene & $0.40(0.24)$ & $0.25(0.28)$ & 2.60 & $0.011^{*}$ \\
$t$ & -1.53 & -2.14 & & \\
$p$ & 0.135 & $0.039^{*}$ & & \\
\hline
\end{tabular}

$\overline{\text { Note. Comparison of Older Adult Group and Young Adult Group was conducted using the Student's }}$ $t$ test and comparison of Hazard prediction scene and Hazard scene was conducted using the paired t test.

${ }^{*} p<.05$. $* * p<.01$.

Table 3 Multivariate Logistic Regression Analysis for Brake Operation of Older Adult Group in Hazard Prediction Scene.

\begin{tabular}{lrll}
\hline Variable & OR & \multicolumn{1}{c}{$95 \% \mathrm{CI}$} & \multicolumn{1}{c}{$p$} \\
\hline Age & 0.93 & $0.82-1.05$ & 0.247 \\
Gender & 0.61 & $0.12-3.23$ & 0.564 \\
PSR & 484.2 & $2.82-83066.59$ & $0.019^{*}$ \\
\hline
\end{tabular}

Note. $N=43$. PSR $=$ palmar sweating response. $\mathrm{OR}=$ Odds ratio. $\mathrm{CL}$ $=$ Confidence interval. $* p<.05$.

association between brake operation and PSR (OR = 484.2, 95\% CI [2.82, 83066.6], $p=0.02$; Table 3).

\section{Brake Response Latency}

We compared the brake response latencies of participants who were observed to brake in the hazard and hazard prediction scenes (Table 4). In the hazard prediction scene, brake responses in the older adult group demonstrated a latency of $M=3.20$ seconds $(S D=1.51)$ and in the young adult group $M=1.58$ second $(S D=$ $0.79)$; the brake latency of the young group was significantly shorter $(p<0.001)$. Furthermore, in the hazard scene, the older adult group demonstrated a latency of $M$ $=1.26(S D=0.57)$ and the young adult group $M=0.83$ $(S D=0.34)$; the brake latency of the young group was significantly shorter $(p<0.001)$. Both the older adult group ( $p<0.001)$ and the young adult group $(p<0.001)$ showed shorter brake response latencies in the hazard scene versus the hazard prediction scene.

\section{SPR Response Latency}

We compared the SPR response latencies of these participants (Table 4). The SPR response latencies in the hazard prediction scene for the older adult group was $M$ $=3.22$ seconds $(S D=2.22)$ and the young adult group was $M=4.49$ seconds $(S D=2.58)$; the SPR response latency of the older adult group was significantly shorter $(p<0.05)$. In the hazard scene, the older adult group had a latency of $M=2.23$ seconds $(S D=0.87)$ while the young adult group was $M=1.93$ seconds $(S D=1.18)$. Both the older $(p<0.001)$ and young adults $(p<0.001)$ showed shorter latencies in the hazard scene than in the hazard prediction scene.

\section{Discussion}

In the hazard scene, which involved visually identifying a pedestrian running into the path of the vehicle and the sudden movement necessary to avoid this pedestrian, all participants successfully operated their brakes. Their brake and SPR response latencies were significantly shorter than in the hazard prediction scene. These reactions are prompted by different mechanisms than the cognitive processes underlying hazard prediction and may be regarded as automatic responses to HP. However, in the hazard prediction scene, which involved slowing before making a left turn into a narrow alleyway, all young adults operated their brakes consistent with simulation footage, while nearly half (46.5\%) of older adults failed to do so.

In the hazard prediction scene, the poor view into the alleyway where the participant was asked to turn necessitated slow, careful driving. We believe that the lack of brake operation in the hazard prediction scene is related to a decrease in predictive ability, possibly the result of overlooking the hazard due to inattentiveness or slowed visual confirmation. It is possible that the older adult group contained participants who did not predict the possibility of a hazard.

While PSR is thought to decline as one ages [34, 35], Table 2 illustrates that the older adult group had higher PSR volumes in both scenes. Increases in PSR magnitude in this group reflect elevated levels of concentration and tension. We also believe that the significant difference in PSR volume between the operation and non-operation groups represents a difference in hazard prediction abilities. The PSR volume of the operation group surpassed the mean value for the older adult 
Table 4 Brake and Skin Potential Reflex Response Latencies.

\begin{tabular}{|c|c|c|c|c|c|c|}
\hline & & & $\begin{array}{l}\text { Older Adult Group } \\
\qquad M(S D)\end{array}$ & $\begin{array}{c}\text { Young Adult Group } \\
\qquad M(S D)\end{array}$ & $t$ & $p$ \\
\hline \multirow{4}{*}{$\mathbf{A}$} & \multirow{4}{*}{ Brake } & Hazard Prediction Scene & $\begin{array}{c}3.20(1.51) \\
n=23\end{array}$ & $\begin{array}{c}1.58(0.79) \\
n=36\end{array}$ & 5.39 & $0.000 * *$ \\
\hline & & Hazard Scene & $\begin{array}{c}1.26(0.57) \\
n=43\end{array}$ & $\begin{array}{c}0.83(0.34) \\
n=36\end{array}$ & 3.90 & $0.000 * *$ \\
\hline & & $t$ & 7.54 & 5.25 & & \\
\hline & & $p$ & $0.000 * *$ & $0.000 * *$ & & \\
\hline \multirow{4}{*}{ B } & \multirow{4}{*}{ SPR } & Hazard Prediction Scene & $\begin{array}{c}3.22(2.22) \\
n=43\end{array}$ & $\begin{array}{c}4.49(2.58) \\
n=36\end{array}$ & -2.34 & $0.022^{*}$ \\
\hline & & Hazard Scene & $\begin{array}{c}2.23(0.87) \\
n=43\end{array}$ & $\begin{array}{c}1.93(1.18) \\
n=36\end{array}$ & 1.31 & 0.196 \\
\hline & & $t$ & 2.72 & 5.40 & & \\
\hline & & $p$ & $0.008 * *$ & $0.000^{* *}$ & & \\
\hline
\end{tabular}

Notes. Comparison of Older adult group and Young adult group was conducted using the Student's $t$ test and comparison of Hazard prediction scene and Hazard scene was conducted using the paired t test. SPR $=$ Skin potential reflex. $* p<.05 . * * p<.01$.

group, indicating that the cognitive processes of hazard prediction may have elevated PSR in these individuals. Furthermore, in the non-operation group, the PSR volume was lower than the mean value for the entire older adult group, which suggests the absence of a sense of tension in non-operation participants. The results of the multivariate logistic regression analysis indicated that brake operation in the hazard prediction scene was not related to the age or gender of participants but to the PSR caused by the hazard prediction scene.

In literature where PSR is an index, reports involving videos of a truck coming toward participants showed that PSR and sense of tension vary with the distance between their own car and the truck. Specifically, the closer the truck is to the participant, the higher these values become [36]. Previous research using similar driving simulation tests has reported that while a clear increase in PSR is observed in both hazard and hazard prediction scenes, the timing of brake operation and PSR and/or SPR responses in older adults with impaired cognitive function does not correspond with the average responses seen in healthy older adults [33].

In both scenes, the young adult group operated their brakes significantly quicker (Table 4). Studies on the brake operation practices of older adult drivers showed that they tend to leave their foot on the accelerator longer [37] and tend to delay "switching" the foot from the accelerator to the brake pedal [38]. In addition, it is generally accepted that young adults have better visual search ability and faster cognitive processing than older adults [39]. The rapid brake operation in the young adult group in this study align with these findings, and it suggests age-based differences.
We believe measurement of PSR alongside monitoring of brake operation is useful in evaluating the HP ability of older adult drivers using a driving simulator, particularly when evaluating the presence or absence of hazard prediction ability.

\section{Limitations and Future Research}

We could not recruit sufficient participants after controlling for gender ratio. It is possible that the difference in gender and driving experience influenced the driving simulation test outcome. Therefore, future studies should examine the influence of participants' age, sex, and driving experience on driving simulation test performance.

The driving simulator used in this experiment employed footage recorded with a consumer model digital camera and had a limited field of view. Since the simulator steering wheel, accelerator, and brake pedal were not linked to the footage, we could not gather detailed information about the appropriateness of the participants' driving operations. In addition, because standards for PSR, SPR, steering wheel, accelerator, and brake responses have not been predetermined, we would need to collect a large number of samples and establish a range of standard responses to determine which responses would be considered appropriate.

The simulator's capacity to evaluate the HP ability of the driver using biological indicators like PSR and SPR is a one strength though we only used two scenes of footage - one as a hazard prediction scene and the other as a hazard scene. Further research must distinguish which measurements and evaluations are appropriate for 
various scene and formulate testing protocols for driving simulations. In addition, it is necessary to examine in detail in the future how much the PSR, SPR and brake responses observed in this driving simulation test reflect the response when actually driving on the road. Further research is needed to clarify the causal relationship between PSR and SPR responses and actual delays and errors in driving.

\section{Conflicts of Interest}

The authors declare no conflict of interest.

\section{Funding statement}

This research was subsidized by JSPS Grant-in-Aid for Science and Technology JP23300245.

\section{References}

[1] Cabinet Office GoJ. White paper on traffic safety in Japan 2017. 2017.

[2] Anstey KJ, Wood J, Lord S, Walker JG. Cognitive, sensory and physical factors enabling driving safety in older adults. Clin Psychol Rev. 2005; 25(1): 45-65.

[3] Ishimatu K, Miura T, Shinohara K. Age influences visual attention characteristics among accident-free and accidentinvolved drivers. Jpn Psychol Res. 2010; 186-200.

[4] Stutts JC, Stewart JR, Martell C. Cognitive test performance and crash risk in an older driver population. Accid Anal Prev. 1998; 30(3): 337-46.

[5] Freund B, Colgrove LA, Petrakos D, McLeod R. In my car the brake is on the right: pedal errors among older drivers. Accid Anal Prev. 2008; 40(1): 403-9.

[6] Horikawa E, Morizono R, Koga A, Horie J. Elderly driving behavior and cognitive functions. Analysis of License Renewal Course Data. IATSS Res. 2009; 33(1): 18-26.

[7] Devos H, Akinwuntan AE, Nieuwboer A, Truijen S, Tant M, De Weerdt W. Screening for fitness to drive after stroke: a systematic review and meta-analysis. Neurology. 2011; 76(8): 747-56.

[8] Fox GK, Bowden SC, Bashford GM, Smith DS. Alzheimer's disease and driving: prediction and assessment of driving performance. J Am Geriatr Soc. 1997; 45(8): 949-53.

[9] Iverson DJ, Gronseth GS, Reger MA, Classen S, Dubinsky RM, Rizzo M. Practice parameter update: evaluation and management of driving risk in dementia: report of the Quality Standards Subcommittee of the American Academy of Neurology. Neurology. 2010; 74(16): 1316-24.

[10] Galski T, Ehle HT, McDonald MA, Mackevich J. Evaluating fitness to drive after cerebral injury: basic issues and recommendations for medical and legal communities. J Head Trauma Rehabil. 2000; 15(3): 895-908.

[11] Fonda SJ, Wallace RB, Herzog AR. Changes in driving patterns and worsening depressive symptoms among older adults. J Gerontol B Psychol Sci Soc Sci. 2001; 56(6): S343-51.

[12] Marottoli RA, Mendes de Leon CF, Glass TA, Williams CS, Cooney LM, Berkman LF, et al. Driving cessation and increased depressive symptoms: prospective evidence from the New Haven EPESE. Established Populations for Epidemiologic Studies of the Elderly. J Am Geriatr Soc. 1997; 45(2): 202-6.

[13] Mezuk B, Rebok GW. Social integration and social support among older adults following driving cessation. J Gerontol B Psychol Sci Soc Sci. 2008; 63(5): S298-303.

[14] Crundall D, Chapman P, Trawley S, Collins L, van Loon E, Andrews B, et al. Some hazards are more attractive than others: drivers of varying experience respond differently to different types of hazard. Accid Anal Prev. 2012; 45: 600-9.

[15] McKenna FP, Horswill MS, Alexander JL. Does anticipation training affect drivers' risk taking? J Exp Psychol Appl. 2006; 12(1): 1-10.

[16] Horswill MS, Marrington SA, McCullough CM, Wood J, Pachana NA, McWilliam J, et al. The hazard perception ability of older drivers. J Gerontol B Psychol Sci. 2008; 63(4): P212-P8.

[17] Sagberg F, Bjørnskau T. Hazard perception and driving experience among novice drivers. Accident Analysis and Prevention. Accid Anal Prev. 2006; 38(2): 407-14.

[18] Finn P, Bragg BW. Perception of the risk of an accident by young and older drivers. Accid Anal Prev. 1986; 18(4): 289-98.

[19] Kuno Y. Human perspiration: Springfield, Illinois: Charles C. Thomas; 1956.

[20] Crundall D, Chapman P, Phelps N, Underwood G. Eye movements and hazard perception in police pursuit and emergency response driving. J Exp Psychol Appl. 2003; 9(3): 163-74.

[21] Kobayashi M, Tomioka N, Ushiyama Y, Ohhashi T. Arithmetic calculation, deep inspiration or handgrip exercise-mediated pre-operational active palmar sweating responses in humans. Auton Neurosci. 2003; 104(1): 58-65.

[22] Ogawa T. Thermal influence on palmar sweating and mental influence on generalized sweating in man. Jpn J Physiol. 1975; 25(4): 525-36.

[23] Ogawa T, Sugenoya J. Pulsatile sweating and sympathetic sudomotor activity. Jpn J Physiol. 1993; 43(3): 275-89.

[24] Homma S, Nakajima Y, Toma S, Ito T, Shibata T. Intracerebral source localization of mental process-related potentials elicited prior to mental sweating response in humans. Neurosci Lett. 1998; 247(1): 25-8.

[25] Mangina CA, Beuzeron-Mangina JH. Direct electrical stimulation of specific human brain structures and bilateral electrodermal activity. Int J Psychophysiol. 1996; 22(1-2): 1-8.

[26] Farrell MJ, Trevaks D, Taylor NA, McAllen RM. Regional brain responses associated with thermogenic and psychogenic sweating events in humans. J Neurophysiol. 
2015; 114(5): 2578-87.

[27] Learmonth GJ, Ackerly W, Kaplan M. Relationships between palmar skin potential during stress and personality variables. Psychosom Med. 1959; 21(2): 150-7.

[28] Martin I, Venables PH. Mechanisms of palmar skin resistance and skin potential. Psychol Bull. 1966; 65(6): 34757.

[29] Venables PH, Martin I. The relation of palmar sweat gland activity to level of skin potential and conductance. Psychophysiology. 1967; 3(3): 302-11.

[30] Taylor DH. Drivers' galvanic skin response and the risk of accident. Ergonomics. 1964; 7(4): 439-51.

[31] Helander M. Applicability of drivers' electrodermal response to the design of the traffic environment. J Appl Psychol. 1978; 63(4): 481-8.

[32] Kinnear N, Kelly SW, Stradling S, Thomson J. Understanding how drivers learn to anticipate risk on the road: A laboratory experiment of affective anticipation of road hazards. Accid Anal Prev. 2013; 50: 1025-33.

[33] Takahashi R, Kobayashi M, Sasaki T, Yokokawa Y, Momose H, Ohhashi T. Driving simulation test for evaluating hazard perception: Elderly driver response characteristics. Transportation Research Part F: Traffic
Psychology and Behaviour. 2017; 49: 257-70.

[34] Ferrer T, Ramos MJ, Pérez-Sales P, Pérez-Jiménez A, Alvarez E. Sympathetic sudomotor function and aging. Muscle Nerve. 1995; 18(4): 395-401.

[35] Fenske NA, Lober CW. Structural and functional changes of normal aging skin. J Am Acad Dermatol. 1986; 15(4 Pt 1): 571-85.

[36] Zheng R, Yamabe S, Nakano K, Suda Y. Biosignal analysis to assess mental stress in automatic driving of trucks: palmar perspiration and masseter electromyography. Sensors (Basel). 2015; 15(3): 5136-50.

[37] Thompson KR, Johnson AM, Emerson JL, Dawson JD, Boer ER, Rizzo M. Distracted driving in elderly and middle-aged drivers. Accid Anal Prev. 2012; 45: 711-7.

[38] Zhang L, Baldwin K, Munoz B, Munro C, Turano K, Hassan S, et al. Visual and cognitive predictors of performance on brake reaction test: Salisbury eye evaluation driving study. Ophthalmic Epidemiology. 2007; 14(4): 216-22.

[39] Bashore TR, Ridderinkhof KR, van der Molen MW. The decline of cognitive processing speed in old age. Current Directions in Psychological Science. 1997; 6(6): 163-9. 